\title{
An Improved Analytical Model for Vertical Velocity Distribution of Vegetated Channel Flows
}

\author{
Xiaonan Tang \\ Department of Civil Engineering, Xi'an Jiaotong-Liverpool University, Suzhou, China \\ Email: Xiaonan.Tang@liverpool.ac.uk,xiao.tang@xjtlu.edu.cn
}

How to cite this paper: Tang, X. (2019). An Improved Analytical Model for Vertical Velocity Distribution of Vegetated Channel Flows. Journal of Geoscience and Environment Protection, 7, 42-60. https://doi.org/10.4236/gep.2019.74004

Received: March 9, 2019

Accepted: April 15, 2019

Published: April 18, 2019

Copyright $\odot 2019$ by author(s) and Scientific Research Publishing Inc. This work is licensed under the Creative Commons Attribution International License (CC BY 4.0).

http://creativecommons.org/licenses/by/4.0/

Open Access

\begin{abstract}
The existence of vegetation plays an important role to protect the ecosystem and water environment in natural rivers and wetlands, but it alters the velocity field of flow, consequently influencing the transport of pollutant and biomass. As a pre-requisite for the analysis of environmental capacity in a channel, the vertical velocity distribution of flows has attracted much research attention; however, there is yet lack of a good prediction model available. For the channel with submerged vegetation, the vertical velocity distribution in the lower vegetation layer will be different from that in the upper flow layer of non-vegetation. In this paper, after review on the most recent two-layer model proposed by Baptist et al., the author has proposed an improved two-layer analytical model by introducing a different mixing length scale $(\lambda)$. The proposed model is based on the momentum equation of flow with the turbulent eddy viscosity assumed as a linear relationship with the local velocity. The proposed model is compared with the Baptist model for different datasets published in the literature, which shows that the proposed analytical model can improve the vertical velocity distribution prediction well compared with the Baptist model for a range of data. This study reveals that the $\lambda$ is well related with the submergence of vegetation $(\mathrm{H} / \mathrm{h})$, as suggested by $\lambda / h=\beta \sqrt{H / h-1}$. When the constant $\beta$ is taken as $3 / 100$, the proposed model shows good agreement with a wide range of datasets studied: flow depth $(\mathrm{H}) /$ vegetation height $(\mathrm{h})$ in 1.25 to 3.33 , different vegetation densities of $a$ in 1.1 to $18.5 \mathrm{~m}^{-1}$ (a defined as the frontal area of the vegetation per unit volume), and bed slopes in $(1.38-4.0) \times 10^{-3}$.
\end{abstract}

\section{Keywords}

Analytical Model, Vegetated Channel Flow, Submerged Vegetation, Velocity

Distribution, Open Channel Flow 


\section{Introduction}

In many natural rivers and wetlands, various types of vegetation exist like bushes, trees, herbs or any other kinds of plant. Sometime they are deliberately arranged to meet engineering or ecological requirement in river or wetland planning and management. The presence of vegetation will affect the hydrodynamic structure of flow by exerting drag force on the flow. In a river or channel with submerged vegetation, its flow is significantly affected by the discontinuous vegetation drag, resulting in a shear layer generated around the top of vegetation. Many researchers have studied the interaction between vegetation and flow, which includes the flow resistance (Kouwen et al., 1969; Temple, 1986; Stone \& Shen, 2002; Cheng, 2015), velocity, Reynolds stress and turbulence intensity (Nepf, 1999; Nepf \& Vivoni, 2000; Lopez \& Garcia, 2001; Ghisalberti \& Nepf, 2006; Poggi et al., 2004; Stoesser et al., 2010; Rahimi et al., 2018; Tang et al., 2018).

The complexity of vegetated channel flow can be interpreted in multiple mechanisms over the depth of flow (Katul et al., 2011; Nikora et al., 2013; Huai et al., 2014). The multiple mechanisms over different layers of flow can be modelled independently (Carollo et al., 2002; Huai et al., 2014; Tang, 2018a, 2018b; Singh et al., 2019). As a pre-requisite for the analysis in resistance and pollutant mixing process of flow and so on, the vertical velocity distribution in vegetated channel flow has drawn great attention of researchers (e.g. Tsujimoto \& Kitamur, 1990, Shimizu \& Tsujimoto, 1994; Nepf \& Koch, 1999; Ghisalberti \& Nepf, 2004; Kubrak, et al., 2008; Tang \& Knight, 2009; Tang et al., 2010, 2011; Nguyen, 2012; Hao et al., 2014). Many investigators have attempted to predict vertical velocity distribution based on semi-empiricism and/or analytical solution of the momentum equation with closure schemes, in which the modelling of eddy viscosity is to describe the turbulent stresses (Klopstra et al., 1997; Meijer \& Van Velzen, 1999; Defina \& Bixio, 2005; Baptist et al., 2007; Huai, et al., 2009, 2014; Yang \& Choi, 2010; Dimitris \& Panayotis, 2011; Nepf, 2012; Nikora et al., 2013; Tang, 2018a, 2019a; Singh et al., 2019).

In the open-channel flow with single-layer submerged vegetation, a strong moment exchange exits between the fast moving flow in the surface layer and the slowly moving flow in the lower vegetation layer, consequently generating a shear region at the interface near the top of vegetation (Poggi et al., 2004; Nezu \& Sanjou, 2008). The flow structure in this region is governed through the mixing length of eddy and vertical turbulent transport of momentum from the surface flow, with the negligible contribution from the pressure gradient. The effect of the shear length scale can be related to the vegetation height and flow depth (Klopstra et al., 1997; Defina \& Bixio, 2005; Tang, 2018a, 2018b, 2019a; Singh et al., 2019).

A review on the studies on the vertical velocity distribution of flow with submerged rigid vegetation shows that different models are used to predict the vertical velocity distribution through and above submerged vegetation (Tang \& Ali, 
2013; Tang, 2019a). These models are so-called two-layer or three-layer methods. In the layer of submerged vegetation, the analytical model of velocity distribution is based on the concept of mixing-layer analogy of eddy (Klopstra et al., 1997; Meijer \& Van Velzen, 1999; Ghisalberti \& Nepf, 2004; Defina \& Bixio, 2005; Baptist et al., 2007; Huai, et al., 2009, 2014; Yang \& Choi, 2010; Nepf, 2012; Tang, 2018a).

For instance, in the two-layer model by Klopstra et al. (1997) the vertical velocity distribution in the vegetation layer was obtained through the analogy of turbulent shear stress. The turbulent eddy viscosity is approximated as the product of the local velocity $(u)$ and a mixing length $(\lambda)$, which is related to the submergence ratio $(\mathrm{H} / \mathrm{h})$ via an empirical formula, see Figure 1 . This empirical formula of $\lambda$ was obtained by fitting limited experimental data. Afterwards, further studies on this approach were undertaken, which suggested that $\lambda$ can be approximated by $0.0144 \sqrt{H h}$ after more data evaluation (Meijer \& Van Velzen, 1999; Defina \& Bixion, 2005). Similarly, Baptist et al. (2007) established their analytical solution of vertical velocity distribution in the vegetation layer, and they suggested that $\lambda$ is approximated by $0.05(H-h)$. Recently, Nepf (2012) proposed an alternative model based on some empirical equations to describe the vertical velocity distribution.

In the evaluation of the above models and other models, Tang \& Ali (2013) and Tang (2019a) showed that the predicted velocity distribution by the above mentioned models is acceptable only for certain experimental datasets, but they do not agree with other datasets. In addition, the predictions by these models show significant differences even for the same dataset. This is not surprising because different constants in their models of $\lambda$ were used. Moreover, none of these models appear to predict well against a wide range of data tested (Tang \& Ali, 2013; Tang, 2018b).

In the present paper, firstly review and discussion is carrying out on the most recent two-layer analytical model by Baptist et al. (2007). Then, based on the mixing length concept of turbulent eddy, the author proposed an alternative improved two-layer model for predicting the vertical velocity distribution of flow with submerged rigid vegetation. In the proposed model, because the submergence of $H / h$ has impact on the eddy size in the mixing region near the top of vegetation, a mixing length scale $(\lambda)$ in the proposed model is proposed to relate to both the height of vegetation and the flow depth, i.e. in a dimensionless formula of

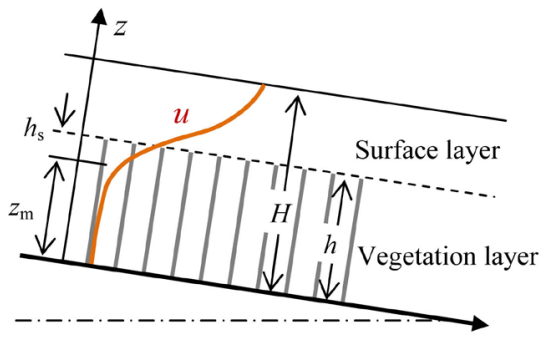

Figure 1. Vertical velocity distribution of flow through submerged vegetation. 
$\lambda / h=\beta \sqrt{H / h-1}$, where $\beta$ is a constant. Finally, a wide range of experimental data from the literature were used to evaluate the proposed analytical model along with the model by Baptist et al. (2007). It was found that when $\beta=3 / 100$ the proposed model agrees well with a range of datasets, which are given by Dunn et al. (1996), Meijer \& Van Velzen (1999), Nguyen (2012) and Hao et al. (2014). However, the Baptist model only suits for certain cases with lower submergence and vegetation density.

\section{Analytical Model by Baptist et al. (2007)}

\subsection{Description of the Analytical Model}

For the channel flow with vegetation, the bed and wall boundary stress are both assumed to be negligible compared with the drag force on the vegetation. Under the steady uniform flow, the governing momentum equation for fully-developed $1-\mathrm{D}$ vegetated flow can be described by

$$
\frac{\partial \tau}{\partial z}=F_{v}-\rho g S_{o}
$$

where $\tau$ is the shear stress, $\rho$ the density of water, $g$ the gravity, $z$ the vertical coordinate above the bed, $S_{o}$ the bed slope and $F_{V}$ is the drag force per unit weight generated by the vegetation, see Figure 1 . The drag force $F_{V}$ is given by:

$$
F_{v}=\left\{\begin{array}{ll}
0.5 \rho C_{D} a u^{2}, & z \leq h \\
0, & z>h
\end{array} ; a=m A_{v}\right.
$$

where $u$ is the stream wise time-averaged velocity, $h$ is the height of vegetation, $C_{D}$ is the drag coefficient, $a$ is the density of vegetation, i.e. the frontal area of vegetation $\left(A_{v}\right)$ per unit volume.

To solve the momentum Equation (1) analytically, Baptist et al. (2007) introduced Boussinesq's eddy viscosity concept to approximate the turbulent shear stress $(\tau)$ as follows:

$$
\tau(z)=\rho v_{t} \frac{\partial u}{\partial z}=\rho\left(c_{p} l u\right) \frac{\partial u}{\partial z}
$$

where $v_{t}$ is the total eddy viscosity of vegetated flow, $l$ is the length scale of vegetation, and $c_{p}$ is the parameter of turbulence intensity.

If the length scale $(l)$ and the turbulence intensity parameter $\left(c_{p}\right)$ are assumed constant within the vegetation, Equation (1) becomes

$$
\left(c_{p} l\right) \frac{\partial^{2}\left(u^{2}\right)}{\partial z^{2}}-C_{D} a u^{2}+2 g S_{o}=0
$$

For given density (a) of vegetation and drag coefficient $\left(C_{D}\right)$, an analytical solution for $u^{2}$ in Equation (4) can be obtained:

$$
u=\sqrt{u_{0}^{2}+a_{v} \exp \left(\frac{z}{L}\right)+b_{v} \exp \left(-\frac{z}{L}\right)}
$$

where $a_{v}$ and $b_{V}$ are the integration constants, which can be determined by appropriate boundary conditions, and 


$$
\begin{gathered}
u_{o}=\left.u\right|_{z=0}=\sqrt{\frac{2 g S_{o}}{a C_{D}}} \\
L=\sqrt{\frac{c_{p} l}{a C_{D}}}
\end{gathered}
$$

Afterwards, by ignoring the last term in the right hand of Equation (5), Baptist et al. (2007) simplified Equation (5) to Equation (8):

$$
u=\sqrt{u_{0}^{2}+a_{v} \exp \left(\frac{z}{L}\right)}
$$

where $a_{\mathrm{v}}$ is described by the following expression:

$$
a_{v}=\frac{2 g S_{o}(H-h) L}{\left(c_{p} l\right) \exp (h / L)}
$$

Equation (8) describes the velocity profile in the vegetation layer.

For the surface layer above the vegetation (i.e. non-vegetation layer), where the velocity can be described by the well-known logarithmic profile, as used by Klopstra et al. (1997), Nepf \& Vivoni (2000), Tang (2018a):

$$
u=\frac{u_{*}}{\kappa} \ln \left(\frac{z-z_{m}}{z_{o}}\right)=\frac{u_{*}}{\kappa} \ln \left[\frac{z-\left(h-h_{s}\right)}{z_{o}}\right]
$$

where $\kappa$ is von Karman's constant $(0.40), Z_{m}\left(=h-h_{s}\right)$ is the zero-plane displacement of the logarithmic profile, $h_{s}$ is the distance from the top of vegetation to the virtual bed of the surface layer (see Figure 1), $z_{o}$ is the equivalent height of bed roughness, and $u_{*}$ is the shear velocity, given by $u_{*}=\sqrt{g S_{o}(H-h)}$.

Furthermore, $h_{s}$ and $z_{o}$ are expressed respectively by:

$$
\begin{gathered}
h_{s}=L(1-\exp (-h / L)) \\
z_{o}=h_{s} \mathrm{e}^{-\kappa} \sqrt{\frac{2 L}{c_{p} l}\left(1+\frac{L}{H-h}\right)}
\end{gathered}
$$

Baptist et al. (2007) validated their method using the data of Nepf \& Vivoni (2000) and recommended that $c_{p} l=(H-h) / 20$.

\subsection{Discussions on the Analytical Model}

Baptist et al. (2007) proposed a simplified analytical model of velocity profile, which mainly aims to introduce an equation for calculating flow resistance in a channel with vegetation. This will help to evaluate the roughness and modelling. However, some assumptions have been made in the development of their analytical model, where some aspects of the assumptions are open for further discussions, which are given as follows:

In the abovementioned analytical model, the analytical solution (8) is an approximated, simplified solution to the full solution (5) by ignoring the term of $\left[b_{v} \exp (-z / L)\right]$, which is usually not zero because $b_{v}$ is not zero. When applying the boundary condition at the top of the vegetation $(z=h)$, where the boundary shear stress is given by $\left.u_{*}\right|_{z=h}=\rho g(H-h) S_{o}$, one can obtain $b_{v}=-a_{v}$ There- 
fore, the solution of Equation (5) may result in some errors, which could not be negligible in certain cases. Thus, it is expected that the solution of Equation (8) may over-predict the velocity near the channel bed in the vegetation layer.

Moreover, at the bed $(z=0)$ of vegetated flow, where the bed shear stress is neglected compared with the drag force of vegetation, the flow velocity can be described by $u_{o}$, given by Equation (6), based on the local equilibrium between gravity force and vegetation drag. However, the solution of Equation (8) does not lead to $u_{o}$ when $z$ approaches to zero, because $a_{v} \exp (z / L)$ becomes $a_{v}$ which is usually not zero but a small limit value. In this respect, the approximate solution of Equation (8) may not properly represent the velocity profile very close to the bed, thus consequently affecting the vertical velocity distribution in both layers.

Finally, since only limited data were used for the calibration of the parameters ( $c_{p}$ and $I$ ) in the model of Baptist et al. (2007), the recommended relationship of $c_{p} l=(H-h) / 20$ may not suit for a wide range of data.

Based on the discussion above, a full solution, rather than a simplified solution, to the governing Equation (1) should be developed in the next section.

\section{Improved Analytical Model}

In this paper, like Klopstra et al. (1997), Defina \& Bixio (2005) and Tang (2018a), the turbulent shear stress $(\tau)$ in Equation ( 1 ) is approximated by Boussinesq hypothesis through a mixing length concept as:

$$
\tau=\rho v_{t} \frac{\partial u}{\partial z}=\rho(\lambda u) \frac{\partial u}{\partial z}
$$

where $\lambda$ is a mixing length of eddy.

For steady flow, inserting Equations (2) \& (13) into (1) produces:

$$
\lambda \frac{\partial^{2}\left(u^{2}\right)}{\partial z^{2}}-C_{D} a u^{2}+2 g S_{o}=0
$$

If vegetation density $(a)$, drag coefficient $\left(C_{D}\right)$ and the mixing length $(\lambda)$ are given, Equation (14) becomes an ordinary differential equation in terms of $u^{2}$. The solution of $u^{2}$ can be obtained in certain boundary conditions. The boundary conditions are considered as follows:

At the bed $(z=0)$, where the bed shear stress is negligible in comparison to the drag force of vegetation, the local equilibrium between vegetation drag and gravity force leads to:

$$
u_{o}=\left.u\right|_{z=0}=\sqrt{\frac{2 g S_{o}}{a C_{D}}}
$$

At the top of vegetation $(z=h)$, the turbulent shear stress is given by:

$$
\left.\tau\right|_{z=h}=\rho g(H-h) S_{o}
$$

Based on the boundary conditions of Equations (15) \& (16), the solution of Equation (15) to $u$ gives the velocity in the vegetation layer, as expressed by 


$$
u=u_{o} \sqrt{1+\eta\left(\frac{H}{h}-1\right) \frac{\sinh \left[\eta\left(\frac{z}{h}\right)\right]}{\cosh (\eta)}} ; \eta=\sqrt{\frac{a C_{D}}{\lambda / h}}
$$

where $\lambda / h$ is related to the submergence $H / h$. In this study, it is assumed as $\beta \sqrt{H / h-1}$, where $\beta$ is a constant.

The velocity of $u$ in Equation (17) will become $u_{o}$ when $z$ approaches to zero (i.e. close to the bed) because $\sinh (\eta z / h)=0$ for $z=0$. Therefore, the solution of Equation (17) does not have such an issue in the Baptist model.

For the surface layer (i.e. non-vegetation layer), the velocity takes the same form as Equation (10), where the unknown parameters $h_{s}$ and $z_{o}$ can be obtained by applying the continuity conditions of the velocity and velocity gradient (or the shear stress) at the interface (i.e. at $z=h$ ) between the surface layer and the vegetation layer. Thus, $h_{s}$ and $z_{o}$ can respectively be described as:

$$
\begin{gathered}
h_{s}=\frac{g S_{o}+\sqrt{\left(g S_{o}\right)^{2}+4\left(\kappa \partial u /\left.\partial z\right|_{z=h}\right)^{2} g S_{o}(H-h)}}{2\left(\kappa \partial u /\left.\partial z\right|_{z=h}\right)^{2}} \\
z_{o}=h_{s} \mathrm{e}^{-\kappa\left(\frac{\left.u\right|_{z=h}}{u_{*}}\right)}
\end{gathered}
$$

\section{Data Used for This Study}

To evaluate both the proposed new model and the Baptist model describe above, the author used a range of different experimental data of submerged rigid vegetation published in the literature. A total of 17 datasets used in this study covers various submergence $(H / h)$, which is 1.25 - 3.33, the vegetation densities $a=1.1$ - $18.5 \mathrm{~m}^{-1}$, and bed slope $S_{o}$ ranging from $1.38 \times 10^{-3}$ to $4 \times 10^{-3}$. The summary of the datasets are shown in Table 1, where the experimental data of Nguyen (2012) include various vegetation densities and different diameter sizes of vegetation. The values of $C_{D}$ in the dataset of emergent vegetation were taken from the original papers if available or assumed according to White (1974) if not.

\section{Results and Discussion}

\subsection{Results}

As shown in Section 3, the characteristic length $\lambda$ reflects the mixing strength of eddy of submerged vegetative flow. The $\lambda$ is closely affected by the submergence $(H / h)$, as recommended as $\beta \sqrt{H / h-1}$ in the present study. The larger $\beta$ value, the bigger $\lambda$ becomes, i.e. the stronger the eddy near the top of vegetation. This implies that the velocity deceases as increasing $\beta$, as demonstrated in Figure 2 as an example. Figure 2 demonstrates that the value of $\beta$ has much greater impact on the predictive velocity in the surface layer than in the vegetation layer. Especially, the velocity close to the channel bed is almost not affected by the constant $\beta$. In other words, $\lambda$ will have great influence on the velocity distribution in the upper region of depth. 


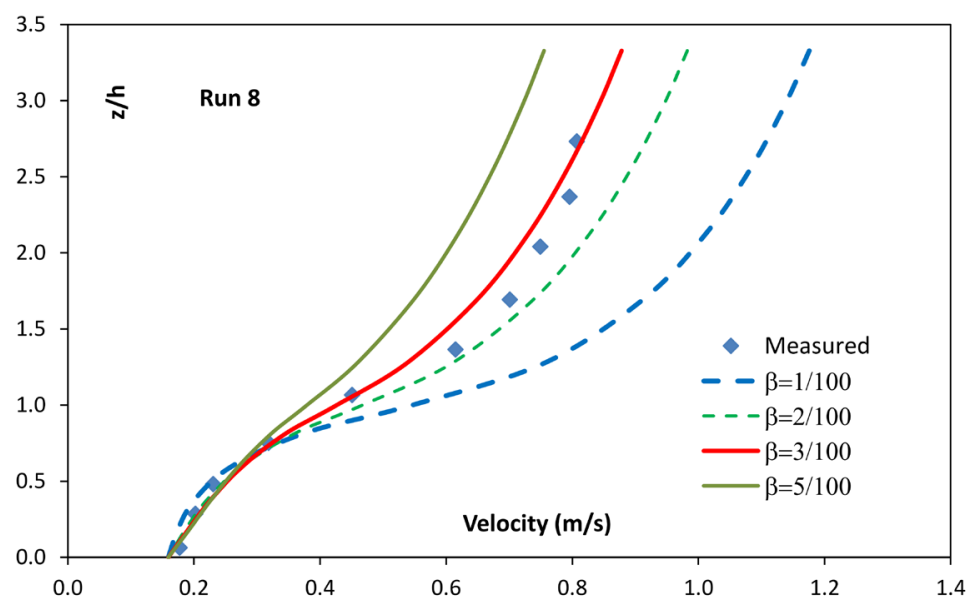

Figure 2. The effect of $\beta$ on the vertical velocity distribution for the data of Dunn et al. (1996).

Table 1. The dataset used for evaluating the models of submerged rigid vegetation.

\begin{tabular}{|c|c|c|c|c|c|c|c|}
\hline Authors & Run & $H \quad(\mathrm{~m})$ & $H / h$ & $a\left(\mathrm{~m}^{-1}\right)$ & $C_{D}$ & $S_{o}$ & $a h$ \\
\hline \multirow[t]{2}{*}{ Dunn et al. (1996) } & 8 & 0.391 & 3.33 & 2.46 & 1.13 & 0.0036 & 0.289 \\
\hline & 9 & 0.214 & 1.82 & 2.46 & 1.13 & 0.0036 & 0.289 \\
\hline \multirow[t]{3}{*}{ Meijer \& Van Velzen (1999) } & 22 & 2.08 & 2.31 & 2.048 & 0.97 & 0.00138 & 1.843 \\
\hline & 34 & 0.99 & 2.20 & 2.048 & 0.97 & 0.0016 & 0.922 \\
\hline & 36 & 1.50 & 3.33 & 2.048 & 0.97 & 0.0014 & 0.922 \\
\hline \multirow[t]{2}{*}{ Lopez \& Garcia (2001) } & 01 & 0.335 & 2.79 & 1.09 & 1.13 & 0.0036 & 0.131 \\
\hline & 09 & 0.214 & 1.78 & 2.49 & 1.13 & 0.0036 & 0.299 \\
\hline \multirow[t]{2}{*}{ Huai et al. (2009) } & R1 & 0.291 & 1.53 & 1.2 & 1.00 & 0.0004 & 0.228 \\
\hline & $\mathrm{R} 2$ & 0.383 & 2.02 & 1.2 & 1.00 & 0.0004 & 0.228 \\
\hline \multirow[t]{6}{*}{ Nguyen (2012) } & A60-15 & 0.15 & 1.5 & 1.78 & 1.13 & 0.004 & 0.178 \\
\hline & A30-15 & 0.15 & 1.5 & 7.11 & 1.13 & 0.004 & 0.711 \\
\hline & B60-15 & 0.15 & 1.5 & 3.67 & 1.13 & 0.004 & 0.367 \\
\hline & B30-15 & 0.15 & 1.5 & 14.67 & 1.13 & 0.004 & 1.467 \\
\hline & $\mathrm{C} 60-15$ & 0.15 & 1.5 & 4.61 & 1.13 & 0.004 & 0.461 \\
\hline & C $30-15$ & 0.15 & 1.5 & 18.44 & 1.13 & 0.004 & 1.844 \\
\hline \multirow[t]{2}{*}{ Hao et al. (2014) } & Test 1 & 0.10 & 1.25 & 1.355 & 1.13 & 0.0035 & 0.108 \\
\hline & Test 2 & 0.11 & 1.38 & 1.355 & 1.13 & 0.004 & 0.108 \\
\hline
\end{tabular}

After extensive tests on $\beta$ values in the proposed $\lambda$ model, the optimal value of $\beta=3 / 100$ was found for the proposed analytical model of $u$. This value of $\beta$ is used in the subsequent figures.

For the simplicity, in the subsequent figures, the model denotes the proposed new model with $\beta=3 / 100$ in this study, whereas the Baptist model denotes the model proposed by Baptist et al. (2007), as described in Section 2. 
Figures 3-7 show the results of comparison between the models (both the new and Baptist models) and the data for the cases of the submergence of $H / h \geq$ 1.5. The comparison shows that the predicted velocity profiles by the new model generally agree well with all the experimental data tested, whereas the Baptist model does not predict well for most the cases when the flows have relatively high submergence (e.g. $H / h \gg 2$ ), see Figure 3(a), Figure 4(c), Figure 5(a) and Figure 6(b). In these high submergence cases, the Baptist model significantly under-predicts the velocity in the surface layer, indicating that the length scale $\left(c_{p} l\right)$ is over-estimated because of a large constant of $(H-h)$ used in the model.

Note that in Figure 7 for the case of Nguyen (2012), both models agree the data reasonably well when the density of vegetation is not too high (e.g. $a h<1.8$ ) under moderate submergence $(H / h=1.5)$. However, if the density of vegetation is very large, e.g. $a h \gg 1.8$ in case $\mathrm{C} 30-15$, it appears that the predictive velocities by the two models are over-estimated in both layers, which indicates the stronger mixing of eddy due to the high density of vegetation. In such a case, because the wake effect between the vegetation of finite height becomes strong, it is expected that the drag coefficient $\left(C_{D}\right)$ will be affected by the density and arrangement formation of vegetation. Thus, for the case of C30 - 15, as the drag

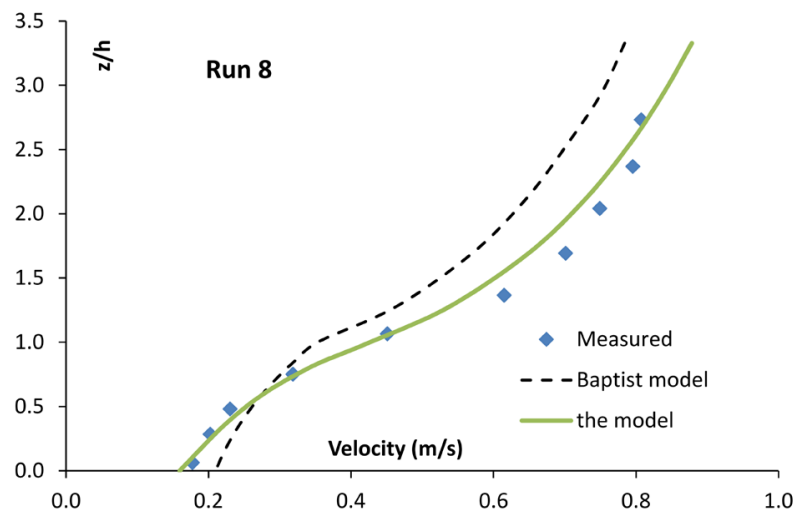

(a)

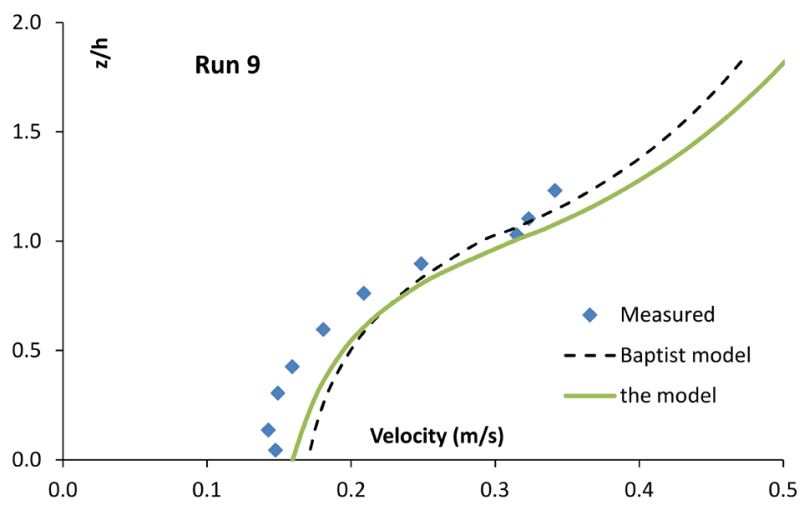

(b)

Figure 3. Comparison for the data by Dunn et al. (1996). (a) $H / h=3.33$, $a h=0.289$; (b) $H / h=1.82, a h=0.289$. 


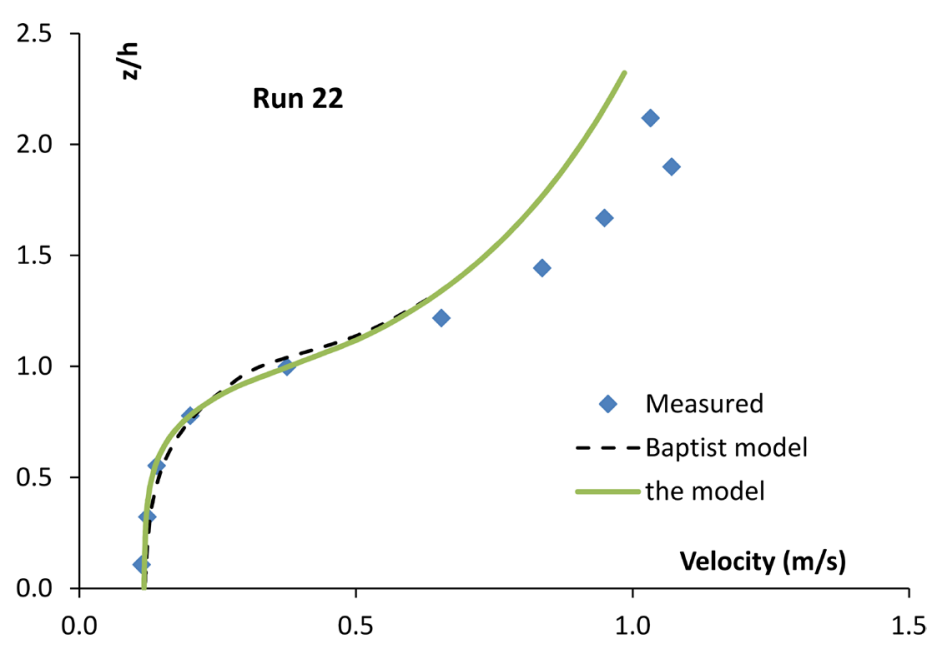

(a)

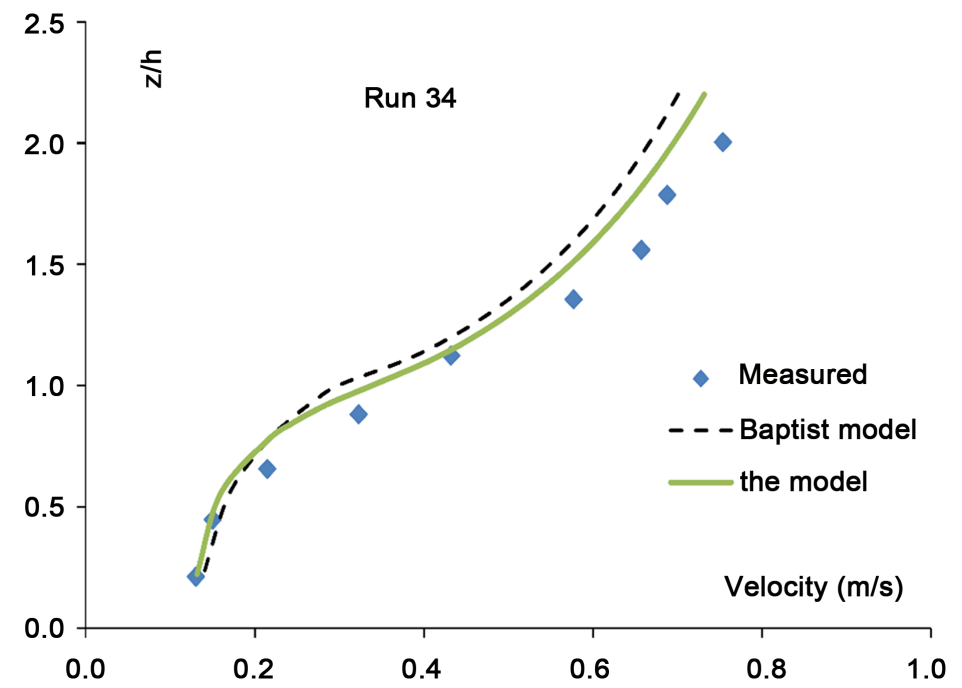

(b)

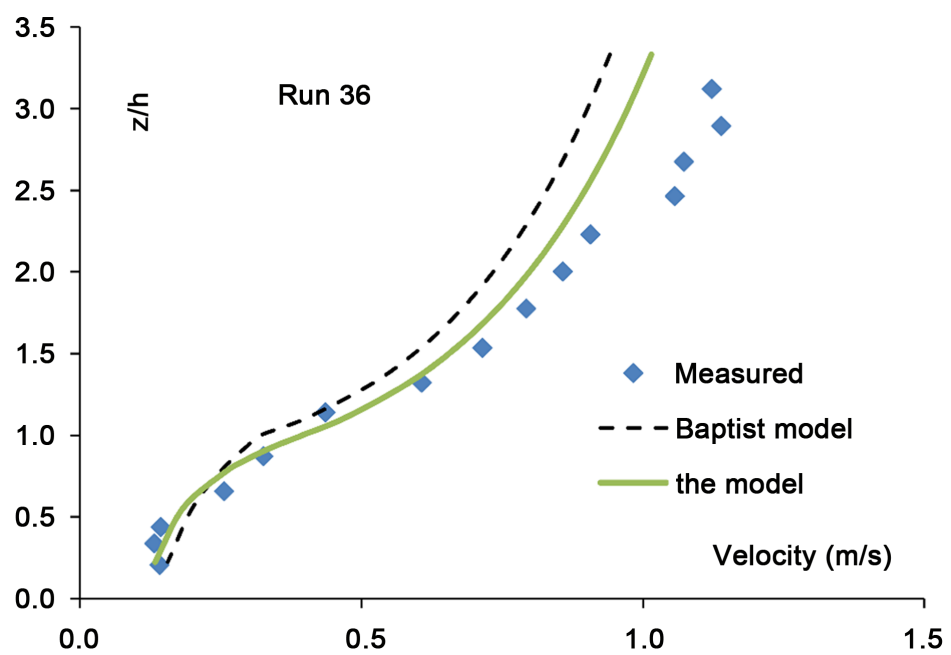

(c)

Figure 4. Comparison for the data by Meijer \& Van Velzen (1999). (a) $H / h=2.31, a h=$ 1.843; (b) $H / h=2.22, a h=0.922$; (c) $H / h=3.33, a h=0.922$. 


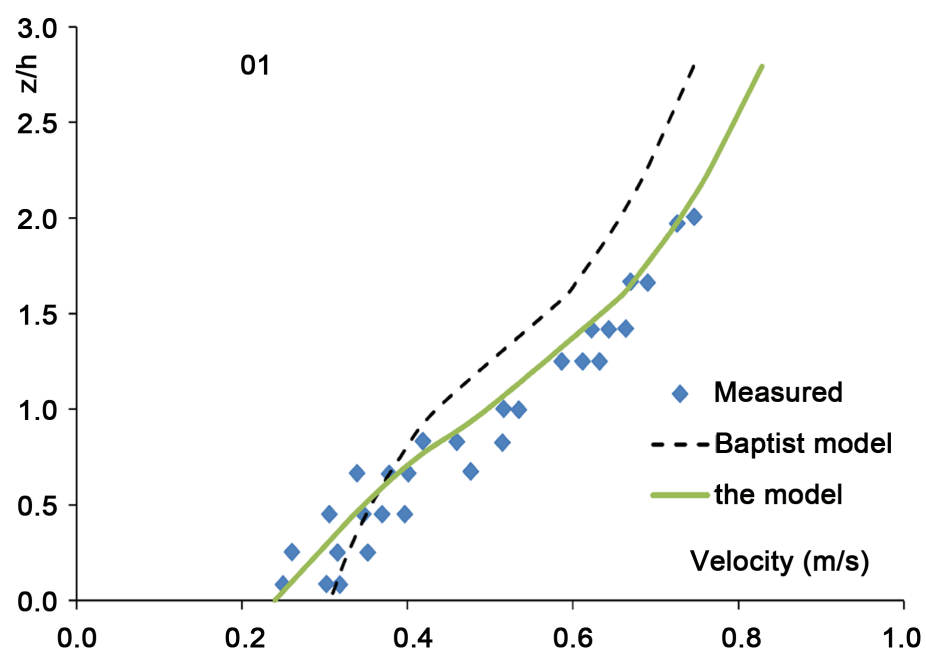

(a)

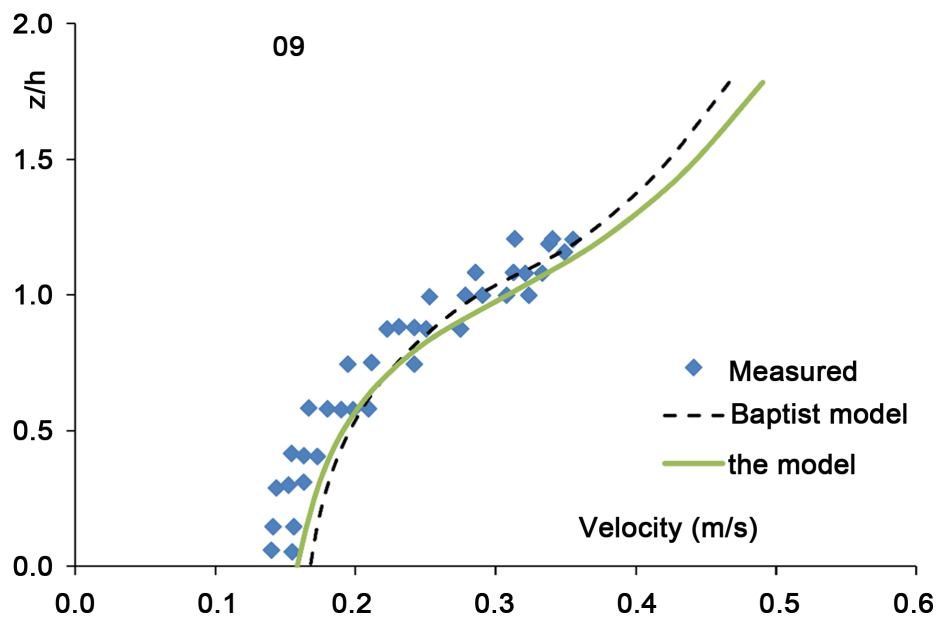

(b)

Figure 5. Comparison for the experimental data by Lopez \& Garcia (2001). (a) $H / h=$ 2.79, $a h=0.131$; (b) $H / h=1.78, a h=0.299$.

coefficient is supposed to increase and assumed to be 1.8, then the new model predicts the velocity reasonably well against the data, see Figure 8 , while the Baptist model does not.

In the case of very shallow submergence as $H / h \ll 1.5$ defined by Nepf (2012), e.g. Hao et al. data in this study, both models under-predict the velocity in the vegetation layer $(z / h<1)$, see Figure 9. In this case, more care should be paid for any predictive model because the value of ah (i.e. a dimensionless parameter of vegetation density) is close to the suggested limit value (0.1) by Nepf (2012).

Further examination of the measured velocity distribution in the vegetation layer shows that the vertical variation of velocity is small near the bed and increases from certain distance below the top of vegetation. It appears a reflection in the velocity profile occurs below the edge of vegetation, and thus this may indicate a deep penetration due to the turbulent stresses. 


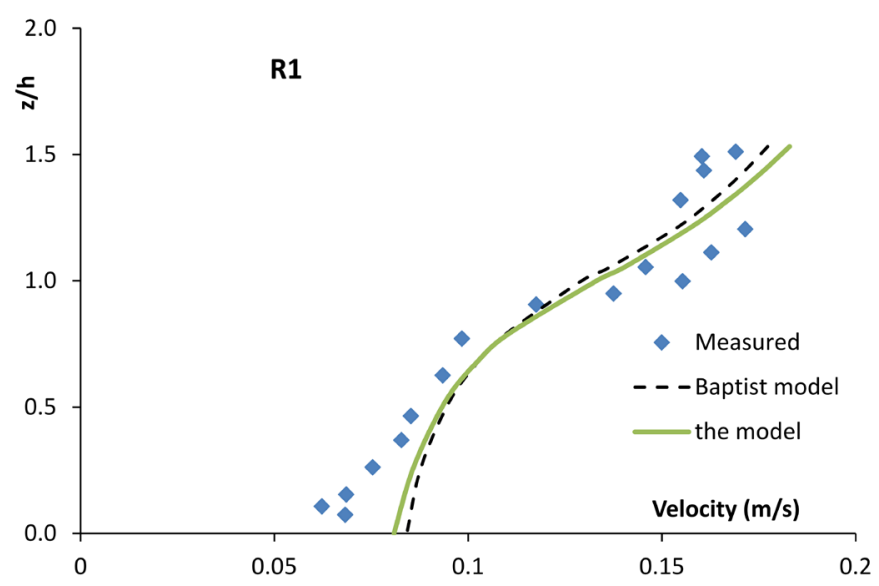

(a)

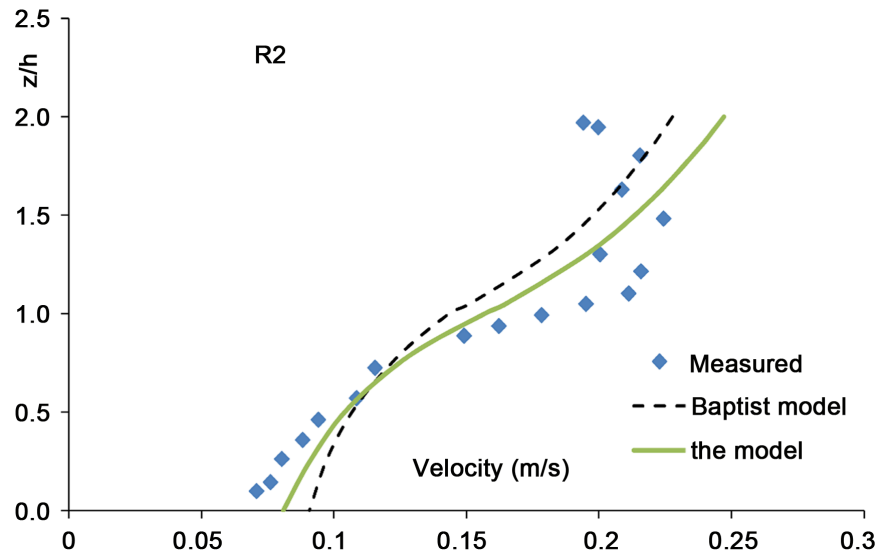

(b)

Figure 6. Comparison for the experimental data by Huai et al. (2009). (a) $H / h=1.53, a h$ $=0.228$; (b) $H / h=2.02$, $a h=0.228$.

\subsection{Discussion}

In the region close to the bed, the predicted velocity by the Baptist model is often larger than the predictive velocity by the proposed model, see Figures 3-9. This is not surprising as discussed in Section 2.2. This is because the simplified solution (8) is used after a removal of the term $\left[b_{v} \exp (-z / L)\right]$, which is usually a negative value because $b_{v}=-a_{v}$, where $a_{v}$ is always a positive value.

To further check the robustness of the models with the experimental data, error analyses were carried out. MAPE (Mean Absolute Percentage Error) is a measure of the error percentage for predicted values of model against the measured values. These individual differences are called as residuals for the data sample that is used for estimation, and the residuals are known as estimation errors for the sample (Tang, 2017, 2019b).

The percentage of error in predicted discharge of each flow depth is calculated by.

$$
E_{u, i}=\frac{\left|u_{a, i}-u_{e, i}\right|}{u_{e, i}}
$$




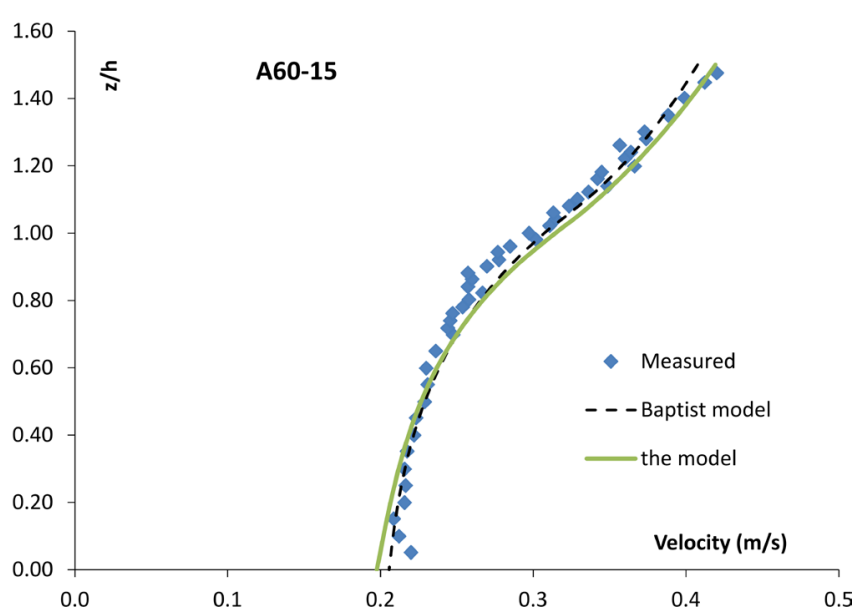

(a)

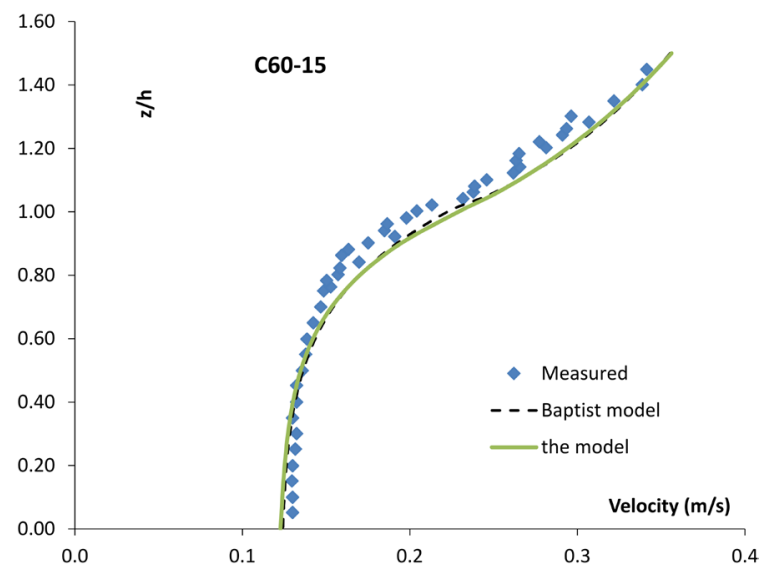

(c)

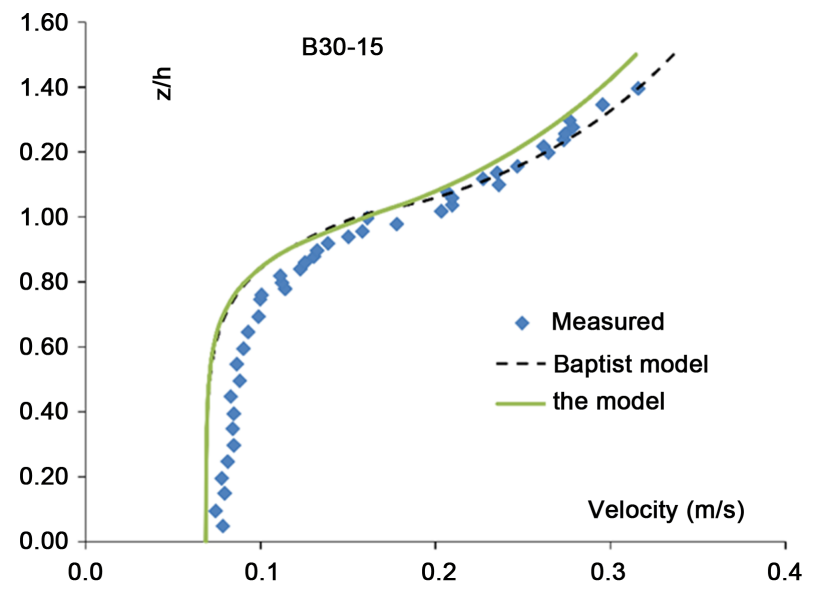

(e)

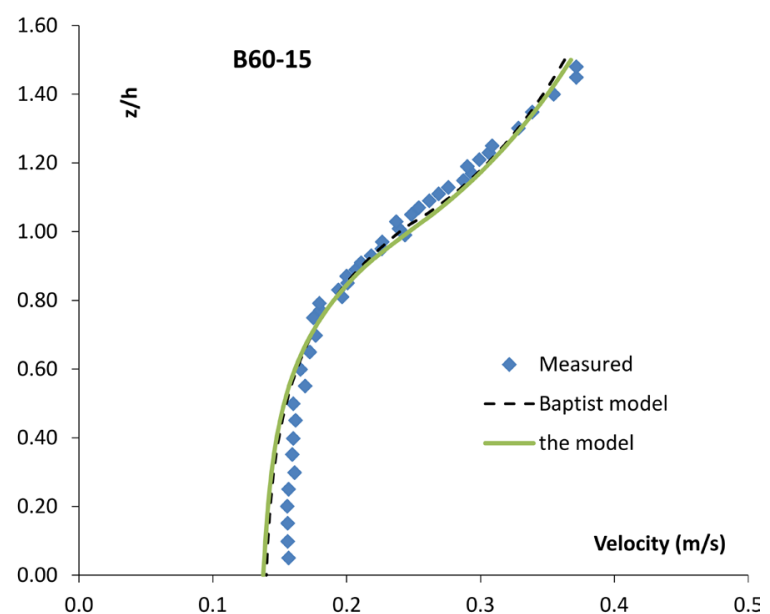

(b)

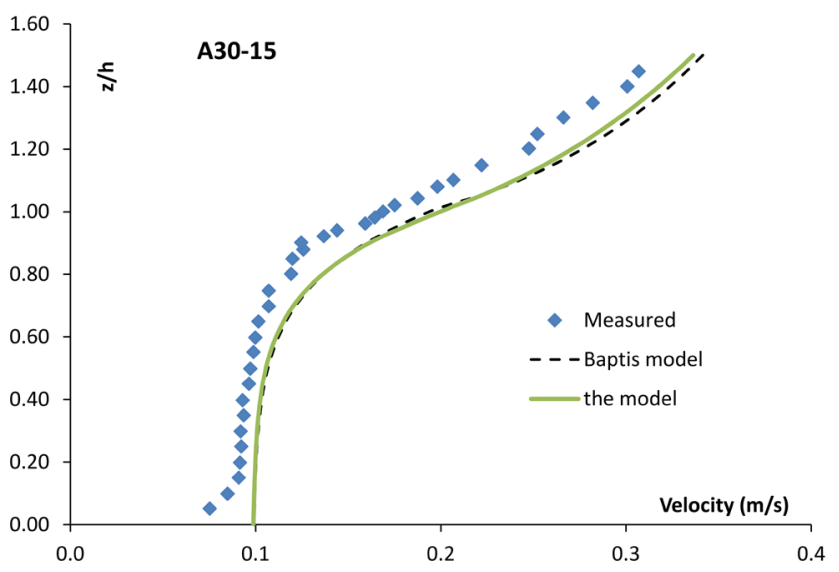

(d)

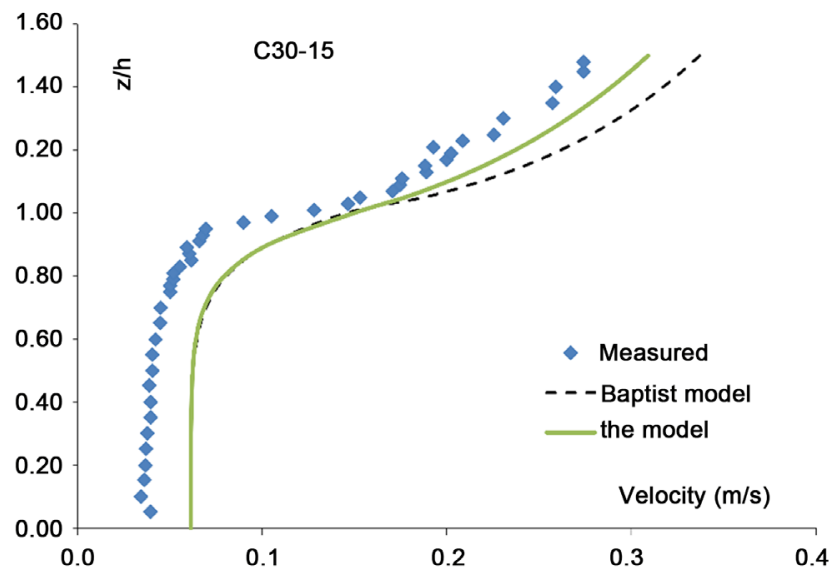

(f)

Figure 7. Comparison for the experimental data by Nguyen (2002). (a) $H / h=1.5, a h=0.178$; (b) $H / h=1.5, a h=0.367$; (c) $H / h=$ $1.5, a h=0.461 ;$ (d) $H / h=1.5, a h=0.711$; (e) $H / h=1.5, a h=1.467$; (f) $H / h=1.5, a h=1.844$.

where $E_{u, i}$ is the error percentage of predicted velocity, and $u_{a, i}$ and $u_{e, i}$ are the predicted and observed velocity at $\mathrm{i}$-th flow depth, respectively. Therefore, the average error percentage of model for an experiment can be calculated by 


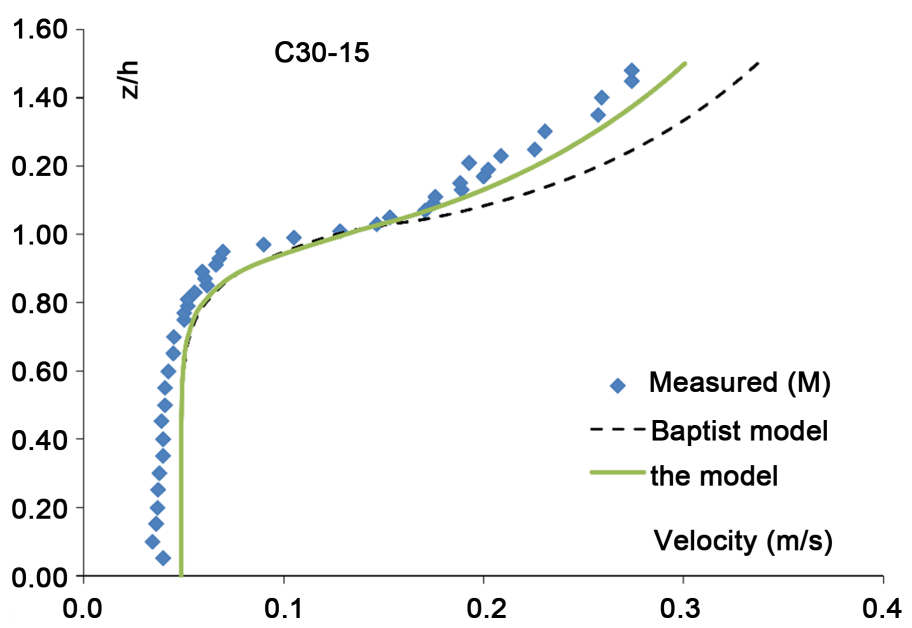

Figure 8. Comparison for the experimental data by Nguyen (2002) for C30-15 using $C_{D}=$ 1.8 .

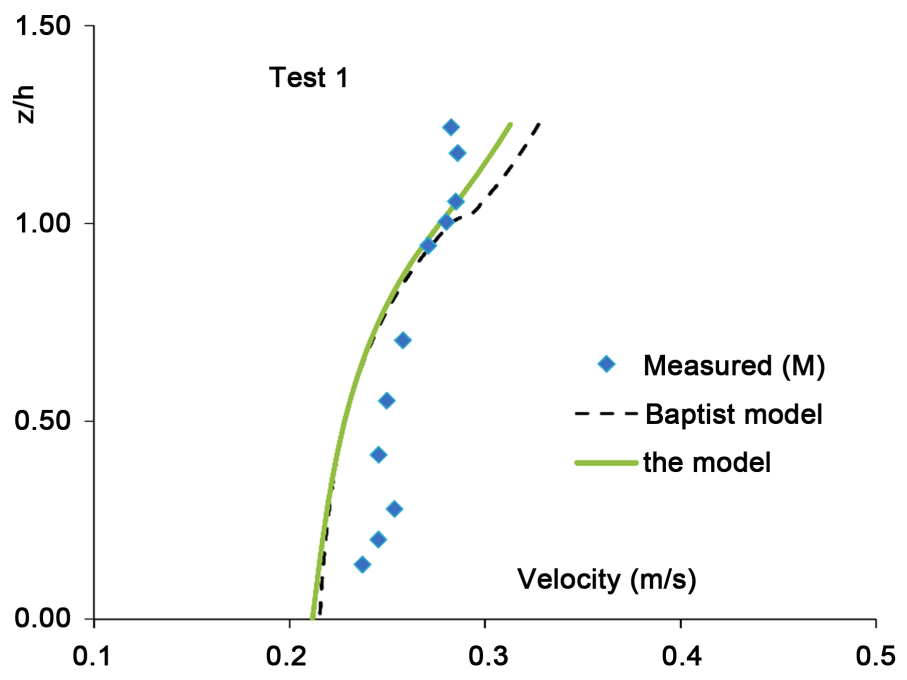

(a)

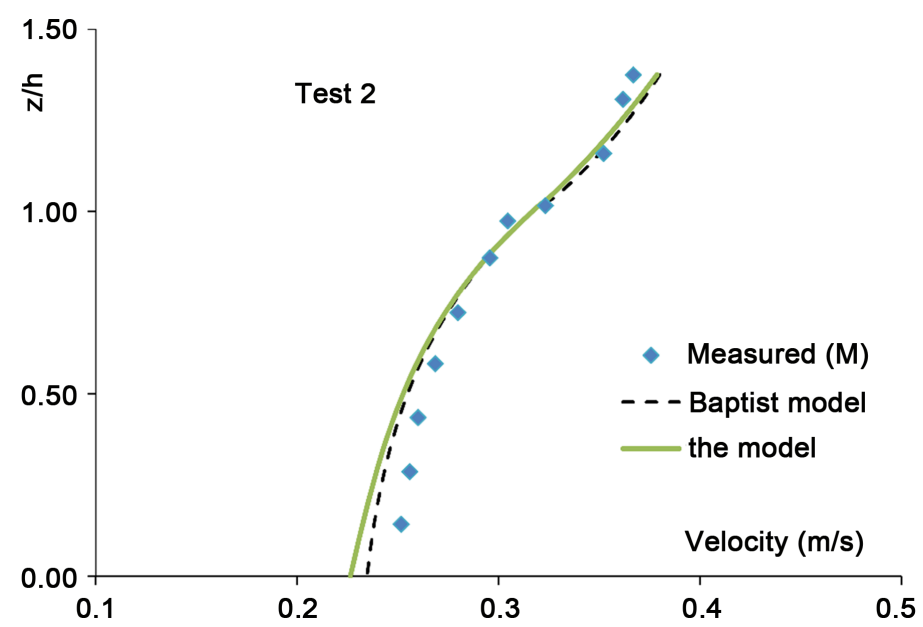

(b)

Figure 9. Comparison for the experimental data by Hao et al. (2014). (a) $H / h=1.25$, $a h=$ 0.108 ; (b) $H / h=1.38, a h=0.108$. 


$$
E_{u}=\frac{1}{N} \sum_{i=1}^{N}\left(E_{u, i}\right)
$$

The values $\left(E_{u}\right)$ of MAPE for all datasets are shown in Table 2, which shows that the proposed model has less the averaged percentage error (9\%) than the Baptist model (about 11.1\%).

As demonstrated in this study, the proposed model with $\lambda / h$ taken as $\beta \sqrt{H / h-1}$ shows good agreement with a range of the data tested, whereas the Baptist model does not agree well for most of the data when $H / h$ is very high (e.g. $H / h \gg 2.0$ ). The reason may be due to the fact that the simplified solution of Equation (8) is used in the Baptist model. When the $3^{\text {rd }}$ term in the full solution of Equation (5) is ignored, the velocity will be over-predicted, consequently affecting the flow velocity in the upper layer (i.e. over-predicted). Another reason may be due to only the flow depth $(H-h)$ of free flow used for the evaluation of mixing length parameter $\left(c_{p} l\right)$. For the cases with higher $H / h(\gg 2)$, the value of $(H-h)$ can be relatively large, which could result in a large value of $c_{p} l$. Thus, $a_{V}$ in Equation (9) is under-valued. Therefore, the predicted velocity from Equation (8) becomes smaller. As a result, the velocity by the Baptist model is under-predicted for higher $H / h$ cases, see Figure 3(a), Figure 4(c) \& Figure 5(a). In the improved analytical model, the analytical solution of Equation (17) is a full solution to Equation (15). $H / h$ is also directly considered in both the solution Equation (17) and the evaluation of mixing length $\lambda$. In other words, the proposed model has considered the impact of $\mathrm{H} / \mathrm{h}$ on the prediction of velocity.

Nevertheless, further study may need to establish the recommended value of constant $\beta=3 / 100$ for even a wide range of data in the future.

Table 2. The MAPE errors (in percentage) of models for the dataset.

\begin{tabular}{cccc}
\hline Experiment & Run & This model & Baptist model \\
\hline Dunn et al. (1996) & 8 & 0.0427 & 0.1485 \\
Meijer \& Van Velzen (1999) & 9 & 0.1263 & 0.1314 \\
Lopez \& Garcia (2001) & 22 & 0.0787 & 0.1069 \\
Huai et al. (2009) & 34 & 0.0817 & 0.1150 \\
& 36 & 0.1005 & 0.1662 \\
Nguyen (2012) & 9 & 0.0749 & 0.1179 \\
& R1 & 0.1047 & 0.1047 \\
& R2 & 0.1106 & 0.1231 \\
& A60-15 & 0.1150 & 0.1497 \\
& A30-15 & 0.0293 & 0.0252 \\
All averaged & B60-15 & 0.1351 & 0.1483 \\
Hao et al. (2014) & B30-15 & 0.0393 & 0.0375 \\
& C60-15 & 0.1345 & 0.1126 \\
& C30-15 & 0.0530 & 0.0591 \\
& Test 1 & 0.1714 & 0.2252 \\
& Test 2 & 0.0688 & 0.0816 \\
& & 0.0318 & 0.0257 \\
& & 0.0907 & 0.1105 \\
\hline
\end{tabular}




\section{Conclusion}

In the open-channel flow of submerged vegetation, the additional resistance of vegetation drag will significantly affect the vertical velocity distribution within the vegetation, which differentiates from that in the upper non-vegetation region. Based on Boussinesq approximation for the mixing length of eddy in the momentum equation, an analytical solution of velocity can be obtained as Equation (17), which describes the velocity profile in the vegetation region. Equation (17) meets the $u_{o}$ near the bed while Equation (8) of the Baptist model does not meet. Through tests against a range of experimental datasets, it was found that the proposed model can predict the velocity reasonably well against a wide range of data for the cases of moderate submergence $(H / h \geq 1.5)$ when the normalized mixing length $(\lambda / h)$ is approximated by $\beta \sqrt{H / h-1}$ with $\beta$ having the optimal value of $3 / 100$. In comparison, the Baptist model does not predict well for the cases when the submergence $(H / h)$ is high (e.g. $\gg 2$ ).

The MAPE error analysis shows that the proposed model has overall less error compared with the Baptist model.

\section{Acknowledgements}

The author would like to thank the support by the National Natural Science Foundation of China (11772270) and the Research Development Funding (RDF-15-01-10) and Key Programme Special Fund (KSF-E-17) of XJTLU.

\section{Conflicts of Interest}

The author declares no conflicts of interest regarding the publication of this paper.

\section{References}

Baptist, M. J., Babovic, V., Rodriguez Uthubrubu, J., Keijzer, M., Uittenbogaadr, R. E., Mynett, A., \& Verwey, A. (2007). On Inducing Equations for Vegetation Resistance. Journal of Hydraulic Research, 45, 435-450. https://doi.org/10.1080/00221686.2007.9521778

Carollo, F. G., Ferro, V., \& Termini, D. (2002). Flow Velocity measurements in Vegetated Channels. Journal of HydraulicEngineering, 128, 664-673. https://doi.org/10.1061/(ASCE)0733-9429(2002)128:7(664)

Cheng, N. (2015). Single-Layer Model for Average Flow Velocity with Submerged Rigid Cylinders. Journal of HydraulicEngineering, 140, Article ID: 06015012. https://doi.org/10.1061/(ASCE)HY.1943-7900.0001037

Defina, A., \& Bixio, A. C. (2005). Mean Flow and Turbulence in Vegetated Open Channel Flow. Water Resources Research, 41, W07006. https://doi.org/10.1029/2004WR003475

Dimitris, S., \& Panayotis, P. (2011). Macroscopic Turbulence Models and Their Application in Turbulent Vegetated Flows. Journal of Hydraulic Engineering, 137, 315-332. https://doi.org/10.1061/(ASCE)HY.1943-7900.0000307

Dunn, C., Lopez, F., \& Garcia, M. (1996). Mean Flow and Turbulence Structure Induced by Vegetation: Experiments. Hydraulic Engineering, Series 51, Urbana, IL: Department 
of Civil Eng., University of Illinois at Urbana-Champaign.

Ghisalberti, M., \& Nepf, H. M. (2004). The Limited Growth of Vegetated Shear Layers. Water Resources Research, 40, w07502. https://doi.org/10.1029/2003WR002776

Ghisalberti, M., \& Nepf, H. M. (2006). The Structure of the Shear Layer in Flows over Rigid and Flexible Canopies. Environmental Fluid Mechanics, 6, 277-301. https://doi.org/10.1007/s10652-006-0002-4

Hao, W. L., Zhu, C. J., \& Chang, X. P. (2014). Research on Vertical Distribution of Longitudinal Velocity in the Flow with Submerged Vegetation. Journal of Heibei University of Engineering, 31, 64-67. (In Chinese)

Huai, W. X., Chen, Z., \& Han, J. (2009). Mathematical Model for the Flow with Submerged and Emerged Rigid Vegetation. Journal of Hydrodynamics, 21, 722-729. https://doi.org/10.1016/S1001-6058(08)60205-X

Huai, W., Wang, W., Hu, Y., Zeng, Y., \& Yang, Z. (2014). Analytical Model of Mean Velocity Distribution in an Open Channel with Double-Layered Rigid Vegetation. Advances in Water Resources, 69, 106-113. https://doi.org/10.1016/j.advwatres.2014.04.001

Katul, G. G., Poggi, D., \& Ridolfi, L. (2011). A Flow Resistance Model for Assessing the Impact of Vegetation on Flood Routing Mechanics. Water Resources Research, 47, W08533.

Klopstra, D., Barneveld, H. J., Noortwijk, J. M., \& Velzen, E. H. (1997). Analytical Model for Hydraulic Roughness of Submerged Vegetation. In Proceeding of 27th Congress of $I A H R$, Theme $A$ (pp. 775-780). New-York: American Society of Civil Engineers (ASCE).

Kouwen, N., Unny, T. E., \& Hill, H. M. (1969). Flow Retardance in Vegetated Channels. Journal of the Irrigation and Drainage Division, 95, 329-342.

Kubrak, E., Kubrak, J., \& Rowinski, P. M. (2008). An Experimental Study of Flow through Rigid Vegetation. Hydrological Sciences Journal, 53, 905-920. https://doi.org/10.1623/hysj.53.4.905

Lopez, F., \& Garcia, M. H. (2001). Mean Flow and Turbulence Structure of Open-Channel Flow through Non-Emergent Vegetation. Journal of Hydraulic Engineering, 127, 392-402. https://doi.org/10.1061/(ASCE)0733-9429(2001)127:5(392)

Meijer, D. G., \& Van Velzen, E. H. (1999). Prototype-Scaleflume Experiments on Hydraulic Roughness of Submerged Vegetation. In 28th International IAHR Conference (1-7). Delft: IAHR.

Nepf, H. M. (2012). Flow and Transport in Regions with Aquatic Vegetation. Annual Reviews Fluid Mechanics, 44, 123-142. https://doi.org/10.1146/annurev-fluid-120710-101048

Nepf, H. M., \& Koch, E. W. (1999). Vertical Secondary Flows in Submerged Plant-Like Arrays. Limnology and Oceanography, 44, 1072-1080. https://doi.org/10.4319/lo.1999.44.4.1072

Nepf, H. M., \& Vivoni, E. R. (2000). Flow Structure in Depth-Limited, Vegetated Flow. Journal of Geophysical Research, 105, 28547-28557. https://doi.org/10.1029/2000JC900145

Nezu, I., \& Sanjou, M. (2008). Turburence Structure and Coherent Motion in Vegetated Canopy Open-Channel Flows. Journal of Hydro-Environment Research, 2, 62-90. https://doi.org/10.1016/j.jher.2008.05.003

Nguyen, H. T. (2012). Characteristics of Hydraulic Resistance and Velocity Profile in Vegetated Open-Channel Flows (pp. 1-201). PhD Thesis, Singapore: Nanyang 
Technological University.

Nikora, N., Nikora, V., \& O’Donoghue, T. (2013). Velocity Profiles in Vegetated Open-Channel Flows: Combined Effects of Multiple Mechanisms. Journal of Hydraulic Engineering, 139, 1021-1032. https://doi.org/10.1061/(ASCE)HY.1943-7900.0000779

Poggi, D., Porporato, A., Ridolfi, L., Albertson, J. D., \& Katul, G. G. (2004). The Effect of Vegetation Density on Canopy Sub-Layer Turbulence. Boundary-Layer Meteorology, 111, 565-587. https://doi.org/10.1023/B:BOUN.0000016576.05621.73

Rahimi, H., Tang, X., \& Wang, X. (2018). Numerical Study on Mixing Layer Vegetation in Open Channel Flow. In Proceedings of the 12th International Symposium on Ecohydraulics (ISE2018) (1-9). Tokyo, Japan.

Shimizu, Y., \& Tsujimoto, T. (1994). Numerical Analysis of Turbulent Open-Channel Flow over a Vegetation Layer Using k-Turbulence Model. Journal of Hydroscience and Hydraulic Engineering, 11, 57-67.

Singh, P., Rahimi, H., \& Tang, X. (2019). Parameterization of the Modeling Variables in Velocity Analytical Solutions of Open-Channel Flows with Double-Layered Vegetation. Environmental Fluid Mechanics, 19, 1-20. https://doi.org/10.1007/s10652-018-09656-8

Stoesser, T., Kim, S. J., \& Diplas, P. (2010). Turbulent Flow through Idealized Emergent Vegetation. Journal of Hydraulic Engineering, 136, 1003-1017. https://doi.org/10.1061/(ASCE)HY.1943-7900.0000153

Stone, B. M., \& Shen, H. T. (2002). Hydraulic Resistance of Flow in Channels with Cylindrical Roughness. Journal of Hydraulic Engineering, 128, 500-506. https://doi.org/10.1061/(ASCE)0733-9429(2002)128:5(500)

Tang, X. (2017). An Improved Method for Predicting Discharge of Homogeneous Compound Channels Based on Energy Concept. Flow Measurement and Instrumentation, 57, 57-63. https://doi.org/10.1016/j.flowmeasinst.2017.08.005

Tang, X. (2018a). A Mixing-Length-Scale-Based Analytical Model for Predicting Velocity Profiles of Open Channel Flows with Submerged Rigid Vegetation. Water and Environment Journal, 32, WEJ12434. https://doi.org/10.1111/wej.12434

Tang, X. (2018b). Methods for Predicting Vertical Velocity Distributions in Open Channel Flows with Submerged Rigid Vegetation. In Proceedings of 21st IAHR-APD Congress (Vol. 1, pp. 567-576). Yagyakart, Indonesia: Universitas Gadjah Mada

Tang, X. (2019a). Evaluating Two-Layer Models for Velocity Profiles in Open-Channels with Submerged Vegetation. Journal of Geoscience and Environment Protection, 7, 68-80. https://doi.org/10.4236/gep.2019.71006

Tang, X. (2019b). A New Apparent Shear Stress-Based Approach for Predicting Discharge in Uniformly Roughened Compound Channels. Flow Measurement and Instrumentation, 65, 280-287. https://doi.org/10.1016/j.flowmeasinst.2019.01.012

Tang, X., \& Ali, S. (2013). Evaluation of Methods for Predicting Velocity Profiles in Open Channel Flows with Submerged Rigid Vegetation. In Proceedings of the 35th IAHR World Congress (Vol. 4, B1, pp. 1744-1755). Beijing: Tsinghua University Press.

Tang, X., \& Knight, D. W. (2009). Lateral Distributions of Streamwise Velocity in Compound Channels with Partially Vegetated Flood Plains. Journal of Science in China Series E: Technological Sciences, 52, 3357-3362.

https://doi.org/10.1007/s11431-009-0342-7

Tang, X., Knight, D. W., \& Sterling, M. (2011). Analytical Model of Streamwise Velocity in Vegetated Channels. Proceedings of the Institution of Civil Engineers: Engineering and Computational Mechanics, 164, 91-102. https://doi.org/10.1680/eacm.2011.164.2.91 
Tang, X., Rahimi, H., Singh, P., Wei, Z., Wang, Y., Zhao, Y., \& Lu, Q. (2018). Experimental Study of Open-Channel Flow with Partial Double-Layered Vegetation. In Proceedings of the 1st International Symposiumon Water Resource and Environmental Management (pp. 1-7). London: EDP Sciences,Hamilton House. https://doi.org/10.1051/e3sconf/20198101010

Tang, X., Sterling, M., \& Knight, D. W. (2010). A General Analytical Modelfor Lateral Velocity Distributions in Vegetated Channels. In A. Dittrich, K. Koll et al. (Eds.), River Flow 2010 (Vol. 1, pp. 469-476). BrundesanstaltfurWasserbau.

Temple, D. M. (1986). Velocity Distribution Coefficients for Grass-Lined Channels. Journal of Hydraulic Engineering, 112, 193-205. https://doi.org/10.1061/(ASCE)0733-9429(1986)112:3(193)

Tsujimoto, T., \& Kitamur, T. (1990). Velocity Profile of Flow in Vegetated Bed Channels (pp. 43-55). KHL Progress Report 1, Hydrualic Lab., Kazavava University.

White, F. M. (1974). Viscous Fluid Flow. New York: McGraw-Hill.

Yang, W., \& Choi, S. (2010). A Two-Layer Approach for Depth-Limited Open Channel Flows with Submerged Vegetation. Journal of Hydraulic Research, 48, 466-475.

https://doi.org/10.1080/00221686.2010.491649 